\title{
MINIMUM MODULI OF WEIGHTED COMPOSITION OPERATORS ON ALGEBRAS OF ANALYTIC FUNCTIONS
}

\author{
TAKUYA HoSOKAwa
}

\begin{abstract}
We study the minimum moduli of weighted composition operators on the disk algebra and the space of bounded analytic functions.
\end{abstract}

\section{Introduction}

Let $\mathbf{D}$ be the open unit disk, $\overline{\mathbf{D}}$ its closure and $\mathbf{T}$ the unit circle. Let $H^{\infty}=H^{\infty}(\mathbf{D})$ be the set of all bounded analytic functions on $\mathbf{D}$ and $A$ be the set of all analytic functions bounded on $\mathbf{D}$ and continuous on $\overline{\mathbf{D}}$, called the disc algebra. Then $H^{\infty}$ and $A$ are Banach algebras with the supremum norm

$$
\|f\|_{\infty}=\sup _{z \in \mathbf{D}}|f(z)| .
$$

In this paper, we will deal with the minimum modulus of analytic functions on $\mathbf{D}$ and $\mathbf{T}$. For $f \in H^{\infty}$, the radial limit $f^{*}$ of $f$ is defined almost everywhere on $\mathbf{T}$. We denote that

$$
\|f\|_{-\infty, \mathbf{D}}=\inf _{z \in \mathbf{D}}|f(z)|
$$

and

$$
\|f\|_{-\infty, \mathbf{T}}=\underset{\omega \in \mathbf{T}}{\operatorname{essinf}}\left|f^{*}(\omega)\right| .
$$

Let $S(\mathbf{D})$ be the set of all analytic self-map of D. For $\varphi \in S(\mathbf{D})$, we can define the composition operator $C_{\varphi}$ on $H^{\infty}$ as $C_{\varphi} f=f \circ \varphi$. Moreover, for $u \in H^{\infty}$, we can define the multiplication operator $M_{u}$ on $H^{\infty}$ as $M_{u} f=u f$. Hence the weighted composition operator $u C_{\varphi}$ is the product of $M_{u}$ and $C_{\varphi}$, that is, $u C_{\varphi} f=M_{u} C_{\varphi} f=u f \circ \varphi$.

To define the weighted composition operators on $A$, it is necessary that $\varphi, u \in A$. Denote by $S(\overline{\mathbf{D}})$ the closed unit ball of $A$. If $\varphi \equiv \omega \in \mathbf{T}, \varphi$ is not in

1991 Mathematics Subject Classification. 47B33.

Key words and phrases. Composition operator, injectivity modulus, closed range.

Received September 13, 2005; revised January 16, 2006. 
$S(\mathbf{D})$ but in $S(\overline{\mathbf{D}})$, and $C_{\varphi}$ is the point evaluation at $\omega$ which acts on $A$. We can identify the set of all point evaluations at boundary points with $\mathbf{T}$. By the maximum modulus principle, it is shown that $S(\overline{\mathbf{D}}) \backslash \mathbf{T} \subsetneq S(\mathbf{D})$.

As well known, $\left\|u C_{\varphi}\right\|=\|u\|_{\infty}$ both on $H^{\infty}$ and on $A$. Putting $u \equiv 1$, we have that $\left\|C_{\varphi}\right\|=1$.

Let $X$ and $Y$ be Banach spaces and $T$ be a bounded linear operator from $X$ to $Y$. The operator norm $\|T\|$ of $T$ is the maximum modulus of its image of the closed unit ball $U_{X}=\left\{x \in X:\|x\|_{X} \leq 1\right\}$. In [2], Müller introduced two quantities as the minimum moduli of $T\left(U_{X}\right)$. We can regard $j(T)$ as the minimum modulus of $T\left(U_{X}\right)$ estimating from the outside and $k(T)$ as the minimum modulus estimating from the inside.

Definition 1.1. Let $T$ be a bounded linear operator from $X$ to $Y$.

(i) The injectivity modulus $j(T)$ of $T$ is defined by

$$
j(T)=\inf \left\{\|T x\|_{Y}:\|x\|_{X}=1\right\} .
$$

(ii) The surjectivity modulus $k(T)$ of $T$ is defined by

$$
k(T)=\sup \left\{r \geq 0: T\left(U_{X}\right) \supset r U_{Y}\right\} .
$$

Though the operator norm holds the triangular inequality, neither $j(T)$ nor $k(T)$ hold it. Some properties of $j(T)$ and $k(T)$ are studied in [2].

Proposition 1.2 [2]. Let $T$ be a bounded linear operator from $X$ to $Y$.

(i) Clearly $0 \leq j(T) \leq\|T\|$ and $0 \leq k(T) \leq\|T\|$.

(ii) If $T$ is invertible, then $j(T)=k(T)=\left\|T^{-1}\right\|^{-1}$.

(iii) $j(T)>0$ (this is said that $T$ is bounded below) if and only if $T$ is one-toone and $\operatorname{Ran} T$ is closed.

(iv) $k(T)>0$ if and only if $T$ is onto.

(v) $j(T)=k\left(T^{*}\right)$ and $k(T)=j\left(T^{*}\right)$.

Example 1.3. Let $l^{2}(\mathbf{N})$ be the Hilbert space of square summable one-sided complex sequences.

(i) Let $F$ be the forward shift operator on $l^{2}(\mathbf{N})$. Then $\|F\|=j(F)=1$ but $k(F)=0$.

(ii) Let $B$ be the backward shift operator on $l^{2}(\mathbf{N})$. Then $\|B\|=k(B)=1$ but $j(B)=0$.

\section{Minimum moduli of weighted composition operators on $H^{\infty}$}

In this section we estimate $j\left(u C_{\varphi}\right)$ and $k\left(u C_{\varphi}\right)$ on $H^{\infty}$. First, we concern with the trivial cases. If $u \equiv 0$ or $\varphi \equiv p \in \mathbf{D}$, then $\operatorname{Ran} u C_{\varphi}$ is a zero or one dimensional subspace spanned by $u$. Hence we have the following.

Proposition 2.1. If $u \equiv 0$ or $\varphi \equiv p \in \mathbf{D}$, then $j\left(u C_{\varphi}\right)=k\left(u C_{\varphi}\right)=0$. 
In the sequel, to exclude these cases, we assume that $u \in H^{\infty}$ is not identically zero and $\varphi \in S(\mathbf{D})$ is not constant. Under this assumption, we call $u C_{\varphi}$ nontrivial. We remark that $u C_{\varphi}$ is injective on $H^{\infty}$ if $u C_{\varphi}$ is non-trivial. This fact and (iv) of Proposition 1.2 imply that $k\left(u C_{\varphi}\right)>0$ if and only if $\left(u C_{\varphi}\right)^{-1}$ is bounded on $H^{\infty}$. Then $\varphi$ is an automorphism of $\mathbf{D}$ and $1 / u$ is in $H^{\infty}$, that is, $\|u\|_{-\infty, \mathbf{D}}>0$. Since $\left(u C_{\varphi}\right)^{-1}=M_{1 / v} C_{\varphi^{-1}}$ where $v=u \circ \varphi^{-1}$, we have the following theorem.

THEOREM 2.2. Let $u C_{\varphi}$ be a non-trivial weighted composition operator on $H^{\infty}$. Then $k\left(u C_{\varphi}\right)>0$ if and only if $\|u\|_{-\infty, \mathbf{D}}>0$ and $\varphi$ is an automorphism of D. Moreover, in such cases, $k\left(u C_{\varphi}\right)=j\left(u C_{\varphi}\right)=\|u\|_{-\infty, \mathbf{D}}$.

Considering the special cases of $u \equiv 1$ and $\varphi(z)=z$, we have the following corollary.

Corollary 2.3. Let $u \in H^{\infty}$ and $\varphi \in S(\mathbf{D})$.

(i) $k\left(M_{u}\right)=\|u\|_{-\infty, \mathbf{D}}$

(ii) If $\varphi$ is an automorphism of $\mathbf{D}, k\left(C_{\varphi}\right)=1$. Otherwise, $k\left(C_{\varphi}\right)=0$.

Next we will consider the estimation of $j\left(u C_{\varphi}\right)$. For convenience, we provide some notation.

Definition 2.4. Define that $D_{\delta}(u)=\{z \in \mathbf{D}:|u(z)| \geq \delta\}$.

In [3], Ohno and Takagi have stated their results in terms of Gelfand transformation and Shilov boundary of $H^{\infty}$. Our main theorem is expressed in function theoretic terms. We need the following lemma (see [4] and [5]).

Lemma 2.5. Let $G$ be a subset of $\mathbf{D}$ such that $\bar{G} \supset \mathbf{T}$. Then, for any $f \in H^{\infty}$,

$$
\sup _{z \in G}|f(z)|=\|f\|_{\infty}
$$

Now we can prove the main theorem.

THEOREM 2.6. Let $u C_{\varphi}$ be a non-trivial weighted composition operator on $H^{\infty}$. Then we have

$$
\begin{aligned}
j\left(u C_{\varphi}\right) & =\sup \left\{\delta: \overline{\varphi\left(D_{\delta}(u)\right)} \supset \mathbf{T}\right\} \\
& =\inf _{\omega \in \mathbf{T}} \limsup _{\varphi\left(z_{n}\right) \rightarrow \omega}\left|u\left(z_{n}\right)\right|
\end{aligned}
$$

where we define the supremum in (1) is equal to 0 if such a constant $\delta$ does not exist, and we define also the infimum in (2) is equal to 0 if $\overline{\varphi(\mathbf{D})} \not \supset \mathbf{T}$.

Proof. Let $d$ be the supremum in (1) and $m$ be the infimum in (2). 
First, we will prove that $j\left(u C_{\varphi}\right) \geq d$. We may suppose that $d>0$. Then for any $\delta$ such that $0<\delta<d, \overline{\varphi\left(D_{\delta}(u)\right)} \supset \mathbf{T}$. By Lemma 2.5, for any $f \in H^{\infty}$ such that $\|f\|_{\infty}=1$,

$$
\begin{aligned}
1 & =\sup \left\{|f(z)|: z \in \varphi\left(D_{\delta}(u)\right)\right\} \\
& =\sup \left\{|f(\varphi(z))|: z \in D_{\delta}(u)\right\} \\
& \leq \delta^{-1} \sup \left\{|u(z)||f(\varphi(z))|: z \in D_{\delta}(u)\right\} \\
& \leq \delta^{-1}\left\|u C_{\varphi} f\right\|_{\infty} .
\end{aligned}
$$

Hence we have that $j\left(u C_{\varphi}\right) \geq \delta$. Since $\delta \in(0, d)$ is arbitrary, we have that $j\left(u C_{\varphi}\right) \geq d$.

Conversely, suppose that $j\left(u C_{\varphi}\right)>0$. For any $r$ such that $0<r<j\left(u C_{\varphi}\right)$, we have that $r<\left\|u C_{\varphi} f\right\|_{\infty}$ where $\|f\|_{\infty}=1$. We will show that $\overline{\varphi\left(D_{r}(u)\right)} \supset \mathbf{T}$ by contradiction.

Suppose that there exists $\zeta \in \mathbf{T} \backslash \overline{\varphi\left(D_{r}(u)\right)}$. Put

$$
f_{n}(z)=\left(\frac{z+\zeta}{2}\right)^{n}
$$

Clearly, we can see that $f_{n} \in H^{\infty}$ and $\left\|f_{n}\right\|_{\infty}=1$ for all positive integer $n$. Since $\zeta \in \mathbf{T} \backslash \overline{\varphi\left(D_{r}(u)\right)}$, we have that $\left|f_{1}(\varphi(z))\right|<1$ for any $z \in D_{r}(u)$. For enough large $n$, we can suppose that $\left|f_{n}(\varphi(z))\right|<r\|u\|_{\infty}^{-1}$ for any $z \in D_{r}(u)$. Then we have that for any $z \in D_{r}(u)$,

$$
\left|u C_{\varphi} f_{n}(z)\right|=\left|u(z) f_{n}(\varphi(z))\right|<r \frac{|u(z)|}{\|u\|_{\infty}} \leq r .
$$

On the other hand, for any $z \in \mathbf{D} \backslash D_{r}(u)$,

$$
\left|u C_{\varphi} f_{n}(z)\right| \leq r\left|f_{n}(\varphi(z))\right| \leq r .
$$

Therefore we get $\left\|u C_{\varphi} f_{n}\right\|_{\infty} \leq r$. Hence we conclude that $j\left(u C_{\varphi}\right) \leq r$. This contradicts our assumption. Thus we have that $\overline{\varphi\left(D_{r}(u)\right)} \supset \mathbf{T}$ and then $r \leq d$. Now we get $j\left(u C_{\varphi}\right)=d$.

Next we prove that $d=m$. Suppose that $m>0$. Fix $\varepsilon>0$ such that $m-\varepsilon>0$. Then for all $\omega \in \mathbf{T}$,

$$
\limsup _{\varphi\left(z_{n}\right) \rightarrow \omega}\left|u\left(z_{n}\right)\right| \geq m-\varepsilon .
$$

This means that $\overline{\varphi\left(D_{m-\varepsilon}(u)\right)} \supset \mathbf{T}$. Now we get $d \geq m-\varepsilon$. Since $\varepsilon$ is arbitrary, we have that $d \geq m$.

To complete our proof, we will show that $d \leq m$. Suppose that $d>0$. For $0<\delta<d$, we have that $\overline{\varphi\left(D_{\delta}(u)\right)} \supset \mathbf{T}$. For all $\omega \in \mathbf{T}$, there exists a sequence $\left\{z_{n}\right\} \in D_{\delta}(u)$ such that $\varphi\left(z_{n}\right) \rightarrow \omega$. Moreover we have that 


$$
\limsup _{\varphi\left(z_{n}\right) \rightarrow \omega}\left|u\left(z_{n}\right)\right| \geq \delta .
$$

This implies that $d \leq m$. This completes our proof.

Considering the special cases, we have the following.

Corollary 2.7. Let $u \in H^{\infty}$ and $\varphi \in S(\mathbf{D})$.

(i) $j\left(\underline{\left.M_{u}\right)=\|u\|_{-\infty, \mathbf{T}}}\right.$.

(ii) If $\overline{\varphi(\mathbf{D})} \supset \mathbf{T}$, then $j\left(C_{\varphi}\right)=1$. Otherwise, $j\left(C_{\varphi}\right)=0$.

Next we state the characterization of the closedness of $\operatorname{Ran} u C_{\varphi}$. We denote by $\hat{f}$ the Gelfand transform of $f \in H^{\infty}$. Let $M\left(H^{\infty}\right)$ be the maximal ideal space of $H^{\infty}$. Then the adjoint $C_{\varphi}^{*}$ of $C_{\varphi}$ induces a continuous map $\Phi$ from $M\left(H^{\infty}\right)$ into $M\left(H^{\infty}\right)$. More precisely we can see that $\widehat{C_{\varphi} f}(x)=\hat{f}(\Phi(x))$ for $x \in M\left(H^{\infty}\right)$. Let $S$ be the Shilov boundary of $M\left(H^{\infty}\right)$ and $\Delta_{\delta}(u)=\{x \in S$ : $|\hat{u}(x)| \geq \delta\}$. Hence, combining our result and the result of [3], we get the following corollary.

COROllary 2.8. Let $u \in H^{\infty}$ and $\varphi \in S(\mathbf{D})$. The followings are equivalent;

(i) $\operatorname{Ran} u C_{\varphi}$ is closed in $H^{\infty}$.

(ii) there exists $\delta>0$ such that $\overline{\varphi\left(D_{\delta}(u)\right)} \supset \mathbf{T}$.

(iii) there exists $\delta>0$ such that $\Phi\left(\Delta_{\delta}(u)\right) \supset S$.

Now we give a typical example which shows what affects the estimation of the injectivity modulus.

Example 2.9. Let $u(z)=1-z$. Let $\varphi(z)=z$ and $\psi(z)=z^{2}$. Then $j\left(u C_{\varphi}\right)=0$ and $j\left(u C_{\psi}\right)=\sqrt{2}$.

Proof. Indeed, $j\left(u C_{\varphi}\right)=j\left(M_{u}\right)=\|1-z\|_{-\infty, \mathbf{T}}=0$.

On the other hand, we have that

$$
\begin{aligned}
j\left(u C_{\psi}\right) & =\inf _{\omega \in \mathbf{T}} \max \left\{|1-\zeta|: \zeta^{2}=\omega\right\} \\
& =\inf _{\theta \in[0, \pi]} \max \left\{\left|1-e^{\theta}\right|,\left|1+e^{\theta}\right|\right\}=\sqrt{2}
\end{aligned}
$$

In the last of this section, we give the comparison between some norms and minimum moduli of $C_{\varphi}$ and $M_{u}$. The essential norm $\|T\|_{e}$ of $T$ is the distance from $T$ to the closed ideal of compact operators, that is, $\|T\|_{e}=\inf \{\|T+K\|$ : $K$ is compact $\}$. It is trivial that $T$ is compact if and only if $\|T\|_{e}=0$. It is known that $C_{\varphi}$ is compact on $H^{\infty}$ if and only if $\overline{\varphi(\mathbf{D})} \cap \mathbf{T} \neq \emptyset$. Moreover if $C_{\varphi}$ is not compact on $H^{\infty}$, then $\left\|C_{\varphi}\right\|_{e}=1$ (see [7]). On the other hand, in [6], it is estimated that $\left\|M_{u}\right\|_{e}=\left\|M_{u}\right\|=\|u\|_{\infty}$. Hence we have the following inequalities. 
Corollary 2.10. Let $u \in H^{\infty}$ and $\varphi \in S(\mathbf{D})$.

(i) $0 \leq k\left(C_{\varphi}\right) \leq j\left(C_{\varphi}\right) \leq\left\|C_{\varphi}\right\|_{e} \leq\left\|C_{\varphi}\right\|=1$ and each of these quantities above is zero or one.

(ii) $0 \leq k\left(M_{u}\right) \leq j\left(M_{u}\right) \leq\left\|M_{u}\right\|_{e}=\left\|M_{u}\right\|=\|u\|_{\infty}$.

\section{Minimum moduli of weighted composition operators on $A$}

In this section, we consider weighted composition operators on the disc algebra $A$. We remark that the phenomena observed through the estimation of the minimum moduli of weighted composition operators on $A$ and $H^{\infty}$ are very similar. We can prove the following results in the similar method in the case of $H^{\infty}$. More precisely, we can prove them only in term of the subset of $\mathbf{T}$, without Lemma 2.5. Here we omit the proof.

We start on the trivial cases.

Proposition 3.1. If $u \equiv 0$ or $\varphi \equiv p \in \overline{\mathbf{D}}$, then $j\left(u C_{\varphi}\right)=k\left(u C_{\varphi}\right)=0$.

We suppose that $u C_{\varphi}$ is non-trivial, that is, $u \neq \equiv 0$ and $\varphi \in S(\overline{\mathbf{D}})$ is not constant. If $u C_{\varphi}$ is non-trivial, then $u C_{\varphi}$ is injective on $A$. Hence we can prove the following results as the same way of the cases of $H^{\infty}$.

THEOREM 3.2. Let $u C_{\varphi}$ be a non-trivial weighted composition operator on A. Then $k\left(u C_{\varphi}\right)>0$ if and only if $u$ has no zero on $\overline{\mathbf{D}}$ and $\varphi$ is an automorphism of $\mathbf{D}$. Moreover, in such cases, $k\left(u C_{\varphi}\right)=j\left(u C_{\varphi}\right)=\|u\|_{-\infty, \mathbf{D}}$.

Corollary 3.3. Let $u \in A$ and $\varphi \in S(\overline{\mathbf{D}})$.

(i) $k\left(M_{u}\right)=\|u\|_{-\infty, \mathbf{D}}$.

(ii) If $\varphi$ is an automorphism of $\mathbf{D}, k\left(C_{\varphi}\right)=1$. Otherwise, $k\left(C_{\varphi}\right)=0$.

Next we estimate $j\left(u C_{\varphi}\right)$ on $A$.

Definition 3.4. Denote that $T_{\delta}(u)=\{z \in \mathbf{T}:|u(z)| \geq \delta\}$.

Since $u C_{\varphi}$ is injective, $j\left(u C_{\varphi}\right)>0$ if and only if $\operatorname{Ran} u C_{\varphi}$ is closed in $A$. We can get the following theorem by the similar proof of Theorem 2.6 replacing $D_{\delta}$ by $T_{\delta}$.

THEOREM 3.5. Let $u C_{\varphi}$ be a non-trivial weighted composition operator on A. Then we have

$$
\begin{aligned}
j\left(u C_{\varphi}\right) & =\sup \left\{\delta: \varphi\left(T_{\delta}(u)\right) \supset \mathbf{T}\right\} \\
& =\inf _{\omega \in \mathbf{T}} \sup \{|u(\zeta)|: \varphi(\zeta)=\omega\}
\end{aligned}
$$

where we define the supremum in (3) is equal to 0 if such a constant $\delta$ does not exist, and we define also the infimum in (4) is equal to 0 if $\varphi(\mathbf{T}) \not \supset \mathbf{T}$. 
Corollary 3.6. Let $u \in A$ and $\varphi \in S(\overline{\mathbf{D}})$.

(i) $j\left(M_{u}\right)=\|u\|_{-\infty, \mathbf{T}}$.

(ii) If $\varphi(\mathbf{T}) \supset \mathbf{T}$, then $j\left(C_{\varphi}\right)=1$. Otherwise, $j\left(C_{\varphi}\right)=0$.

\section{REFERENCES}

[1] C. C. Cowen ANd B. D. MacCluer, Composition operators on spaces of analytic functions, CRC Press, Boca Raton, 1995.

[2] V. MÜLLER, Spectral theory of linear operators, Birkhäuser, Basel, 2003.

[3] S. Ohno AND H. TAKagi, Some properties of weighted composition operators on algebras of analytic functions, J. Nonlinear and Convex Analysis 2 (2001), 369-380.

[ 4 ] R. RoAn, Composition operators on $H^{p}$ with dense range, Indiana Univ. Math. J. 27 (1978), 159-162.

[ 5] D. SARASON, Weak-star generators of $H^{\infty}$, Pacific J. Math. 17 (1966), 519-528.

[6] H. Takagi, J. Takahashi and S. UeKi, The essential norms of a weighted composition operator on the ball algebra, Acta. Sci. Math. (Szeged) 70 (2004), 819-829.

[ 7 ] L. Zheng, The essential norms and spectra of composition operators on $H^{\infty}$, Pacific J. Math. 203 (2002), 503-510.

1-10-8 402 Kosugi-JinYaCHO

NAKaHARA, KaWASAKI

KANAGAWA 211-0062

JAPAN

E-mail: turtlemumu@yahoo.co.jp 